Artigo Original

Original Article

Cláudia Giglio de Oliveira Gonçalves ${ }^{1}$ Adriana Bender Moreira Lacerda ${ }^{1}$ Bianca Simone Zeigelboim ${ }^{1}$ Jair Mendes Marques ${ }^{1}$ Débora Luders²

Descritores

Perda auditiva Música

Limiar auditivo Audiometria Saúde do trabalhador

Keywords

Hearing loss

Music

Auditory threshold

Audiometry

Occupational health

Endereço para correspondência: Cláudia Giglio Oliveira Gonçalves R. Felisberto Fiore Dorazio, 146, Santa Felicidade, Curitiba (PR), Brasil, CEP: 82410-460.

E-mail: claudia.goncalves@utp.br

Recebido em: 24/11/2011

\section{Limiares auditivos em músicos militares: convencionais e altas frequências}

\section{Auditory thresholds among military musicians: conventional and high frequency}

\section{RESUMO}

Objetivo: Analisar e caracterizar os limiares auditivos tonais entre $500 \mathrm{~Hz}$ e $16.000 \mathrm{~Hz}$ de músicos profissionais, integrantes de uma banda militar. Métodos: Realizou-se um estudo de coorte histórico, que comparou um grupo de 50 músicos profissionais (banda militar) com um grupo sem histórico de exposição profissional a som intenso (44 sujeitos); e homogêneos ( $<<0,05$ ) em relação ao gênero (masculino) e à idade (em torno de 35 anos). Foram realizados exames de audiometria tonal limiar convencional e de altas frequências (de 9.000 a $16.000 \mathrm{~Hz}$ ). Resultados: A exposição à música desencadeou alterações auditivas permanentes. Quanto aos limiares auditivos convencionais, no grupo exposto encontrou-se $32 \%$ dos músicos com alterações auditivas neurossensoriais e no grupo não exposto encontrou-se 2,27\%. Os sujeitos expostos à música tiveram 14,54 vezes mais risco de adquirir alterações auditivas neurossensoriais do que o grupo não exposto. Após 20 anos de exposição à música foram observadas pioras significativas nos limiares auditivos, principalmente nas altas frequências, e ocorreram diferenças entre os grupos a partir de 30 anos de idade. Conclusão: A exposição à música desencadeou alterações auditivas permanentes, evidenciando diferenças em relação ao grupo não exposto à música, registradas na avaliação audiológica convencional e em altas frequências.

\begin{abstract}
Purpose: To analyze and characterize tonal auditory thresholds between $500 \mathrm{~Hz}$ and $16,000 \mathrm{~Hz}$ of professional musicians, specifically members of a military band. Methods: A historic cohort study was carried out comparing one group of 50 professional musicians (military band) with another group (44 subjects) without history of professional exposure to intense sound; the groups were homogenous $(\mathrm{p}<0.05 \%)$ in relation to gender (male) and age (about 35 years old). Tone threshold audiometry for conventional (from 500 to 8,000 Hz) and high frequencies was applied (from 9,000 to 16,000 Hz). Results: Exposure to music triggered permanent hearing impairment. As to conventional auditory thresholds, the exposed and non-exposed groups presented sensorineural hearing loss of 32 and $2.27 \%$, respectively. The subjects exposed to music had 14.54 times more risks of acquiring sensorineural hearing loss than the non-exposed group. After 20 years of exposure to music, significant worsening was observed in auditory thresholds, especially at extended high frequencies, and differences occurred between the groups from the age of 30 on. Conclusion: Exposure to music caused permanent hearing impairment, showing differences in relation to the non-exposed group, registered in conventional audiometry and at high-frequency audiometry.
\end{abstract}

Trabalho realizado no Programa de Pós-graduação (Mestrado e Doutorado) em Distúrbios da Comunicação, Universidade Tuiutí do Paraná - UTP - Tuiuti (PR), Brasil.

(1) Programa de Pós-graduação (Mestrado e Doutorado) em Distúrbios da Comunicação, Universidade Tuiuti do Paraná - UTP - Tuiuti (PR), Brasil.

(2) Departamento de Fonoaudiologia, Universidade Estadual do Centro-Oeste-UNICENTRO-Tuiuti (PR), Brasil. Conflito de interesse: nada a declarar. 


\section{INTRODUÇÃO}

A literatura mundial há algumas décadas vem estudando a exposição ocupacional ao ruído intenso e definindo os riscos dessa exposição em função dos fatores relacionados ao ruído (intensidade e tempo de exposição) e ao indivíduo exposto (gênero, idade, entre outros).

Já a exposição ocupacional à música iniciou-se a menos tempo do que a exposição ao ruído. Sabe-se que a música tocada por grupos musicais como bandas de rock, pop, jazz, orquestra sinfônica, entre outros, pode atingir níveis elevados de pressão sonora, considerados nocivos para a audição, podendo interferir negativamente na carreira dos músicos.

Estudos realizados com músicos clássicos demonstraram que os níveis de pressão sonora nas orquestras, durante ensaios ou apresentações, podem variar de 77 a $93 \mathrm{~dB}(\mathrm{~A})$, chegando a picos de $110 \mathrm{~dB}(\mathrm{~A})^{(1-3)}$.

Pesquisadores ao redor do mundo descreveram as alterações auditivas provocadas pela exposição à música, como comprometimento auditivo ou diferenças nos limiares auditivos, não necessariamente como alterações próprias de perdas auditivas, principalmente nas frequências altas ${ }^{(3-10)}$.

Para um diagnóstico precoce da alteração auditiva em músicos, evidenciando comprometimentos que ainda não são visíveis na audiometria convencional, alguns estudos vêm utilizando a audiometria de altas frequências (na faixa de $9.000 \mathrm{~Hz}$ a $20.000 \mathrm{~Hz}$ ) juntamente com a audiometria convencional (na faixa de $250 \mathrm{~Hz}$ a $8.000 \mathrm{~Hz})^{(3,8,9,11-13)}$. Esta opção é realizada pelo audiologista, pois a audiometria de altas frequências é um importante exame na detecção de perdas auditivas localizadas na base do ducto coclear, antes mesmo que os seus efeitos característicos apareçam na faixa de frequência convencional ${ }^{(14,15)}$.

Apesar da intensificação dos estudos sobre exposição ocupacional à música, ainda não se tem clareza sobre o início do comprometimento auditivo em relação aos anos de exposição à música e da influência do fator idade sobre os limiares auditivos dos músicos.

O objetivo desse estudo foi analisar e caracterizar os limiares auditivos tonais entre 500 e $16.000 \mathrm{~Hz}$ em músicos profissionais, integrantes de uma banda militar.

\section{MÉTODOS}

Trata-se de um estudo de coorte histórico, que comparou os limiares auditivos de um grupo de músicos profissionais (banda militar) expostos à música diariamente, em ensaios e apresentações, com um grupo similar quanto à idade e gênero, mas sem histórico de exposição profissional a níveis de pressão sonora elevados.

Este estudo obteve aprovação do Comitê de Ética e Pesquisa da UTP sob n ${ }^{\circ} 14 / 08$ e os procedimentos foram realizados após a assinatura do Termo de Consentimento Livre e Esclarecido. Utilizou-se como critério de exclusão sujeitos com comprometimento de orelha média, sendo excluídos dois sujeitos do grupo de músicos.
O grupo de músicos profissionais foi composto por 50 integrantes de uma banda militar no Brasil, expostos a níveis de pressão sonora elevados (música) diariamente (4 horas diárias). A banda é formada por instrumentos de sopro e por percussão, sendo que a maioria são instrumentistas de sopro. Os níveis de pressão sonora avaliados durante os ensaios, por meio de leitura com medidor de pressão sonora integrador, posicionado na frente da banda por quatro horas de ensaio, variou de 90,1 a 110,3 dB(A), com níveis médios de 91 a 96,3 dB(Leq).

Os músicos da banda eram todos do gênero masculino, com idades entre 21 e 51 anos (média de 34,9 anos, desvio padrão de 7,8; mediana=34 anos) e tempo de atuação na banda entre um e 29 anos (média de 14,2 anos e desvio padrão de 7,7). Apesar de serem integrantes de uma banda militar, os músicos ingressaram no exército para exercer a função de músico e não foram submetidos ao treinamento com armamentos.

O grupo sem histórico de exposição ocupacional a níveis de pressão sonora elevados contava com um total de 44 sujeitos, todos do gênero masculino, com idades entre 20 e 51 anos (média de 35,6 anos, desvio padrão de 9,36; mediana=34 anos). Esse grupo foi composto por estudantes e sujeitos de diversas atividades profissionais que não apresentavam níveis de pressão sonora elevados, voluntários, convidados a participarem da pesquisa.

Todos os sujeitos foram submetidos à inspeção do meato acústico externo para descartar-se possíveis obstruções que pudessem comprometer o exame auditivo. Todos os sujeitos estavam em repouso acústico de, no mínimo 14 horas, antes de realizarem a audiometria.

Foram testados os limiares auditivos por audiometria convencional (nas frequências de $500 \mathrm{a} 8.000 \mathrm{~Hz}$ - equipamento audiômetro Interacoustics ${ }^{\circledR}$, modelo AC40 com fones TDH-39, com calibração de acordo com norma I.S.O 389/64) e por audiometria de altas frequências (de 9.000 a $16.000 \mathrm{~Hz}$ - equipamento audiômetro AC40 com fones KOOS/HV PRO e intensidade sonora em decibel nível de audição - dB NA, calibrado segundo o padrão ANSI S3.6/69). O efeito da idade sobre a audição foi controlado para os limiares auditivos tonais aéreos de $500 \mathrm{~Hz}$ a $8.000 \mathrm{~Hz}$, com a utilização da tabela I.S.O 1999*.

O critério de normalidade auditiva foi definido para os limiares auditivos tonais por via aérea em até $25 \mathrm{~dB}$ NA para a faixa de frequência de 500 a $8.000 \mathrm{~Hz}$.

Para a análise dos dados utilizou-se métodos estatísticos que permitissem a determinação dos resultados significativos da avaliação audiológica, considerando-se o nível de significância de 0,05 (5\%). Aplicou-se estatísticas descritivas: o teste t de Student para a comparação dos grupos, o teste de Fisher para testar as correlações entre os exames audiológicos e o teste não paramétrico de MannWhitney na análise dos limiares auditivos tonais convencionais entre os grupos. O teste ANOVA de Kruskal-Wallis foi aplicado para a análise dos limiares tonais auditivos em função do tempo de exposição à música. Para a análise, o tempo de serviço dos músicos em anos foi agrupado em três categorias de dez em dez anos e para as análises considerando-se a idade, agrupo-se em três categorias de dez em dez anos.

\footnotetext{
* Normal air conduction hearing threshold values as a function of age for otologically normal persons according to ISO standard ISO 1999 (1990), obtained from database.A-. The data represent median values for men and women
} 


\section{RESULTADOS}

Os dois grupos são homogêneos em relação à média de idade (teste $t$ de Student $\mathrm{p}=0,8128$, ao nível 0,05 ), ou seja, não houve diferença entre as médias de idades dos dois grupos.

Os resultados da audiometria convencional revelaram a ocorrência de alterações auditivas neurossensoriais em 32\% (16) dos sujeitos do grupo dos músicos profissionais e 2,27\% (1) dos sujeitos do grupo dos não músicos.

$\mathrm{Na}$ população estudada, os sujeitos expostos à música tiveram 14,54 vezes mais risco de adquirir alterações auditivas neurossensoriais do que o grupo não exposto (Risco Relativo=14,54; IC95\% 1,9-100,5) e o excesso de risco atribuível à exposição à música foi estimado em 29 músicos por 100 (Risco Atribuível=0,298).

Os integrantes da banda não referiram exposição extra-ocupacional ao ruído intenso (para esporte ou lazer), mas 23 exercem atividades com música além daquelas desenvolvidas pela banda militar.

Analisou-se os limiares auditivos do grupo exposto à música profissionalmente em função do tempo de exposição em anos (Tabela 1).

Em ambas as orelhas houve diferença nos limiares auditivos de altas frequências (de $9.000 \mathrm{a} 16.000 \mathrm{~Hz}$ ) entre aqueles expostos à música de $1 \mathrm{a} 10$ anos, comparados com aqueles expostos há mais de 21 anos.
$\mathrm{Na}$ frequência de $12.500 \mathrm{~Hz}$ observou-se que, em ambas as orelhas, houve diferença entre os limiares auditivos também entre os expostos à música por 11 a 20 anos com aqueles na faixa de exposição superior a 21 anos. Na orelha esquerda as diferenças nos limiares auditivos entre os tempos de exposição foram também em 10.000 e 14.000 Hz. Já para os limiares auditivos nas frequências convencionais houve diferença apenas em $6.000 \mathrm{~Hz}$ na orelha esquerda entre as faixas de exposição.

Naqueles músicos com 11 a 20 anos de exposição à música, as frequências de $12.500 \mathrm{~Hz}$ (ambas as orelhas), $10.000 \mathrm{~Hz}$ (orelha direita) e $14.000 \mathrm{~Hz}$ (orelha esquerda), tiveram diferenças (ao nível 0,05) em relação àqueles com mais de 21 anos.

Observou-se diferenças nos limiares auditivos para algumas frequências entre os grupos (Tabela 2).

Nas altas frequências, diferenças foram observadas na orelha direita em $9.000 \mathrm{~Hz}$ e bilateralmente em $14.000 \mathrm{~Hz}$ e $16.000 \mathrm{~Hz}$.

Os limiares auditivos convencionais entre os grupos (expostos e não expostos) foram comparados de acordo com a faixa etária, após aplicação da tabela I.S.O 1999 (Tabela 3).

A maioria das frequências, em ambas as orelhas, apresentou diferenças entre os grupos.

Os limiares auditivos entre os grupos (expostos e não expostos) foram comparados em relação às faixas etárias, pela audiometria de altas frequências (Tabela 4).

Tabela 1. Limiares auditivos tonais aéreos dos músicos, por frequências, em função do tempo de exposição profissional à música ( $\mathrm{n}=50$ )

\begin{tabular}{|c|c|c|c|c|c|c|c|}
\hline \multirow{2}{*}{ Orelha } & \multirow{2}{*}{ Frequência $(\mathrm{Hz})$} & \multicolumn{3}{|c|}{ Tempo de exposição (anos) } & \multirow{2}{*}{ Estatística $\mathrm{H}$} & \multirow{2}{*}{ Valor $p$} & \multirow{2}{*}{ Diferenças } \\
\hline & & 1 a 10 & 11 a 20 & 21 ou mais & & & \\
\hline \multirow{13}{*}{ Direita } & 500 & 13 & 24 & 13 & 0,8088 & 0,6674 & - \\
\hline & 1.000 & 13 & 24 & 13 & 2,3229 & 0,3275 & - \\
\hline & 2.000 & 13 & 24 & 13 & 0,5122 & 0,7741 & - \\
\hline & 3.000 & 13 & 24 & 13 & 1,3134 & 0,5186 & - \\
\hline & 4.000 & 13 & 24 & 13 & 0,6967 & 0,7059 & - \\
\hline & 6.000 & 13 & 24 & 13 & 3,6430 & 0,1618 & - \\
\hline & 8.000 & 13 & 24 & 13 & 1,5146 & 0,4689 & - \\
\hline & 9.000 & 13 & 24 & 13 & 6,5341 & $0,0381^{*}$ & $(a) \neq(c)$ \\
\hline & 10.000 & 13 & 24 & 13 & 10,0268 & $0,0066^{\star}$ & $(a) \neq(c)$ \\
\hline & 11.000 & 13 & 24 & 13 & 9,5588 & $0,0084^{\star}$ & $(\mathrm{a}) \neq(\mathrm{c})$ \\
\hline & 12.500 & 13 & 24 & 13 & 10,7432 & $0,0046^{*}$ & $(\mathrm{a}) \neq(\mathrm{c}) ;(\mathrm{b}) \neq(\mathrm{c})$ \\
\hline & 14.000 & 13 & 24 & 13 & 13,2847 & $0,0013^{*}$ & $(a) \neq(c)$ \\
\hline & 16.000 & 13 & 24 & 13 & 13,8569 & $0,0010^{*}$ & $(a) \neq(c)$ \\
\hline \multirow{13}{*}{ Esquerda } & 500 & 13 & 24 & 13 & 2,4601 & 0,2908 & - \\
\hline & 1.000 & 13 & 24 & 13 & 0,9695 & 0,6159 & - \\
\hline & 2.000 & 13 & 24 & 13 & 1,8021 & 0,4061 & - \\
\hline & 3.000 & 13 & 24 & 13 & 2,6808 & 0,2617 & - \\
\hline & 4.000 & 13 & 24 & 13 & 3,5005 & 0,1737 & - \\
\hline & 6.000 & 13 & 24 & 13 & 8,4142 & $0,0149^{\star}$ & $(a) \neq(c)$ \\
\hline & 8.000 & 13 & 24 & 13 & 3,5959 & 0,1656 & - \\
\hline & 9.000 & 13 & 24 & 13 & 6,9201 & $0,0314^{*}$ & $(a) \neq(c)$ \\
\hline & 10.000 & 13 & 24 & 13 & 9,8973 & $0,0071^{*}$ & $(\mathrm{a}) \neq(\mathrm{c}) ;(\mathrm{b}) \neq(\mathrm{c})$ \\
\hline & 11.000 & 13 & 24 & 13 & 10,7437 & $0,0046^{*}$ & $(a) \neq(c)$ \\
\hline & 12.500 & 13 & 24 & 13 & 12,0709 & $0,0024^{*}$ & $(\mathrm{a}) \neq(\mathrm{c}) ;(\mathrm{b}) \neq(\mathrm{c})$ \\
\hline & 14.000 & 13 & 24 & 13 & 12,0487 & $0,0024^{*}$ & $(\mathrm{a}) \neq(\mathrm{c}) ;(\mathrm{b}) \neq(\mathrm{c})$ \\
\hline & 16.000 & 13 & 24 & 13 & 9,3015 & $0,0096^{*}$ & $(\mathrm{a}) \neq(\mathrm{c})$ \\
\hline
\end{tabular}

*Valores significativos ( $p \leq 0,05)$ - Teste ANOVA de Kruskal-Wallis; (a) 1 a 10 anos; (b) 11 a 20 anos; (c) 21 ou mais anos de exposição 
Tabela 2. Demonstrativo das médias e medianas dos limiares auditivos tonais de altas frequências nos grupos ( $n=94)$

\begin{tabular}{|c|c|c|c|c|c|c|c|c|c|c|}
\hline \multirow{2}{*}{ Orelha } & \multirow{2}{*}{$\begin{array}{c}\text { Freq. } \\
(\mathrm{Hz})\end{array}$} & \multicolumn{4}{|c|}{ Expostos } & \multicolumn{4}{|c|}{ Não expostos } & \multirow{2}{*}{ Valor $p$} \\
\hline & & $n$ & Média & Mediana & $\mathrm{DP}$ & $n$ & Média & Mediana & $\mathrm{DP}$ & \\
\hline \multirow{6}{*}{ Direita } & 9.000 & 50 & 21,7 & 20,0 & 21,5 & 44 & 13,1 & 12,5 & 9,8 & $0,016301^{*}$ \\
\hline & 10.000 & 50 & 23,9 & 20,0 & 15,7 & 44 & 20,7 & 20,0 & 14,0 & 0,304369 \\
\hline & 11.200 & 50 & 22,6 & 20,0 & 15,7 & 44 & 19,2 & 15,0 & 16,6 & 0,321766 \\
\hline & 12.500 & 50 & 28,6 & 25,0 & 16,4 & 44 & 28,5 & 20,0 & 20,9 & 0,974020 \\
\hline & 14.000 & 50 & 33,1 & 30,0 & 15,7 & 44 & 22,4 & 15,0 & 18,3 & $0,006109^{*}$ \\
\hline & 16.000 & 50 & 36,8 & 35,0 & 16,2 & 44 & 23,3 & 15,0 & 19,6 & $0,001723^{*}$ \\
\hline \multirow{6}{*}{ Esquerda } & 9.000 & 50 & 18,2 & 15,0 & 14,1 & 44 & 15,1 & 15,0 & 11,0 & 0,244698 \\
\hline & 10.000 & 50 & 20,8 & 20,0 & 16,1 & 44 & 21,0 & 15,0 & 16,0 & 0,946674 \\
\hline & 11.200 & 50 & 21,9 & 20,0 & 16,9 & 44 & 22,0 & 15,0 & 17,6 & 0,967451 \\
\hline & 12.500 & 50 & 27,9 & 25,0 & 13,9 & 44 & 29,1 & 25,0 & 17,6 & 0,735823 \\
\hline & 14.000 & 50 & 32,6 & 30,0 & 18,1 & 44 & 24,5 & 22,5 & 17,0 & $0,040069^{*}$ \\
\hline & 16.000 & 50 & 33,6 & 30,0 & 16,0 & 44 & 25,4 & 20,0 & 18,9 & $0,052710^{*}$ \\
\hline
\end{tabular}

Legenda: DP = desvio-padrão

Tabela 3. Limiares auditivos tonais convencionais médios, após controle da idade pela Norma I.S.O. 1999

\begin{tabular}{|c|c|c|c|c|c|c|c|c|}
\hline \multirow{2}{*}{ Orelha } & \multirow{2}{*}{$\begin{array}{c}\text { Freq. } \\
(\mathrm{Hz})\end{array}$} & \multicolumn{3}{|c|}{ Expostos } & \multicolumn{3}{|c|}{ Não expostos } & \multirow{2}{*}{ Valor $p$} \\
\hline & & $\mathrm{n}$ & Média & $\mathrm{DP}$ & $\mathrm{n}$ & Média & DP & \\
\hline \multirow{7}{*}{ Direita } & 500 & 50 & 8,64 & 7,51 & 44 & 7,41 & 4,40 & 0,3435 \\
\hline & 1000 & 50 & 8,36 & 5,75 & 44 & 7,14 & 4,11 & 0,2440 \\
\hline & 2000 & 50 & 8,12 & 6,28 & 44 & 5,18 & 4,93 & $0,0142^{*}$ \\
\hline & 3000 & 50 & 8,96 & 8,42 & 44 & 5,64 & 5,23 & $0,0261^{*}$ \\
\hline & 4000 & 50 & 11,48 & 9,63 & 44 & 6,25 & 5,49 & $0,0020^{\prime}$ \\
\hline & 6000 & 50 & 10,20 & 8,91 & 44 & 5,68 & 5,53 & $0,0046^{\prime}$ \\
\hline & 8000 & 50 & 9,98 & 11,46 & 44 & 5,82 & 6,99 & 0,0394 \\
\hline \multirow{7}{*}{ Esquerda } & 500 & 50 & 10,46 & 8,29 & 44 & 6,45 & 4,13 & $0,0047^{*}$ \\
\hline & 1000 & 50 & 8,90 & 9,65 & 44 & 7,41 & 4,29 & 0,3467 \\
\hline & 2000 & 50 & 10,38 & 10,01 & 44 & 5,95 & 5,36 & $0,0103^{*}$ \\
\hline & 3000 & 50 & 10,36 & 9,14 & 44 & 5,41 & 4,88 & $0,0018^{\star}$ \\
\hline & 4000 & 50 & 13,56 & 11,55 & 44 & 5,91 & 6,35 & $0,0002^{\prime}$ \\
\hline & 6000 & 50 & 10,94 & 9,76 & 44 & 6,45 & 5,82 & $0,0092^{*}$ \\
\hline & 8000 & 50 & 8,90 & 11,82 & 44 & 5,43 & 6,18 & 0,0840 \\
\hline
\end{tabular}

*Valores significativos ( $\mathrm{p} \leq 0,05)$ - Teste Mann-Whitney

Legenda: DP = desvio-padrão

Para a faixa etária de 20 a 29 anos não ocorreram diferenças (teste de Mann-Whitney) entre os grupos. Já na faixa etária de 30 a 39 anos observou-se diferenças entre os grupos para os limiares auditivos de 14.000 e $16.000 \mathrm{~Hz}$ bilateralmente; e na orelha direita em 2.000, 6.000 e $9.000 \mathrm{~Hz}$. Na faixa etária de 40 a 51 anos de idade, as diferenças entre os grupos foram para os limiares tonais de $14.000 \mathrm{~Hz}$ para a orelha direita e de $16.000 \mathrm{~Hz}$ para a orelha esquerda.

Entre aqueles músicos com limiares auditivos dentro dos padrões de normalidade até $8.000 \mathrm{~Hz}$, houve diferenças significativas em comparação com o grupo não exposto à música para as frequências de $14.000 \mathrm{~Hz}$ e $16.000 \mathrm{~Hz}$ na orelha direita (Tabela 5).

\section{DISCUSSÃO}

Esse estudo analisou a audição de músicos profissionais (50 sujeitos), comparando os limiares auditivos com um grupo de sujeitos não músicos (44 sujeitos) e sem histórico de exposição ocupacional a níveis de pressão sonora elevados, porém homogêneos ( $\mathrm{p}<0,05$ em relação ao gênero (masculino) e média de idades (em torno de 35 anos).

Encontrou-se alterações auditivas (limiares auditivos superiores a $25 \mathrm{~dB} N A$ ) com características de terem sido induzidas por Níveis de Pressão Sonora Elevados (alterações nos limiares auditivos neurossensoriais superiores a $25 \mathrm{~dB} \mathrm{NA} \mathrm{em} \mathrm{3.000,} \mathrm{e/}$ ou 4.000 , e/ou $6.000 \mathrm{~Hz}$ ) entre os músicos (32\%), o que significou um risco de 14,54 vezes maior de desenvolvimento de alteração auditiva do que no grupo sem histórico de exposição à música. A porcentagem de alterações auditivas encontrada é similar ao estudo que avaliou a audição de músicos percussionistas e encontrou $39 \%$ de perdas auditivas e maior proporção de alterações auditivas quando comparado com o grupo controle, que era equivalente em relação ao gênero e idade ${ }^{(16)}$. Em estudo com 329 estudantes de música (idades entre 18 e 25 anos) não se observou perdas auditivas, mas sim entalhes acústicos na audição de $45 \%$ dos estudantes ( $78 \%$ deles apresentavam entalhe acústico em $6.000 \mathrm{~Hz}$ e $22 \%$ em $4.000 \mathrm{~Hz})^{(17)}$. Outros estudos também encontraram alterações auditivas em músicos, mas não 
Tabela 4. Limiares auditivos tonais em altas frequências, de acordo com as faixas etárias, em ambos os grupos ( $\mathrm{n}=94)$

\begin{tabular}{|c|c|c|c|c|c|c|}
\hline Faixas etárias & Orelha & Frequência $(\mathrm{Hz})$ & Expostos & Não expostos & Estatística Z & Valor $p$ \\
\hline \multirow{12}{*}{20 a 29 anos $(n=25)$} & \multirow{6}{*}{ Direita } & 9.000 & 9 & 16 & $-0,375804$ & 0,707063 \\
\hline & & 10.000 & 9 & 16 & $-0,171870$ & 0,863540 \\
\hline & & 11.000 & 9 & 16 & 0,057551 & 0,954106 \\
\hline & & 12.500 & 9 & 16 & $-0,570991$ & 0,568006 \\
\hline & & 14.000 & 9 & 16 & 0,744622 & 0,456501 \\
\hline & & 16.000 & 9 & 16 & 1,023785 & 0,305937 \\
\hline & \multirow{6}{*}{ Esquerda } & 9.000 & 9 & 16 & $-0,633693$ & 0,526282 \\
\hline & & 10.000 & 9 & 16 & $-0,600834$ & 0,547951 \\
\hline & & 11.000 & 9 & 16 & 0,257703 & 0,796636 \\
\hline & & 12.500 & 9 & 16 & $-0,028533$ & 0,977237 \\
\hline & & 14.000 & 9 & 16 & 0,512889 & 0,608029 \\
\hline & & 16.000 & 9 & 16 & 1,513717 & 0,130099 \\
\hline \multirow{12}{*}{30 a 39 anos $(n=39)$} & \multirow{6}{*}{ Direita } & 9.000 & 26 & 13 & 2,536245 & $0,011205^{*}$ \\
\hline & & 10.000 & 26 & 13 & 1,761939 & 0,078081 \\
\hline & & 11.000 & 26 & 13 & 1,789652 & 0,073511 \\
\hline & & 12.500 & 26 & 13 & 2,493148 & $0,012662^{*}$ \\
\hline & & 14.000 & 26 & 13 & 2,917314 & $0,003531^{*}$ \\
\hline & & 16.000 & 26 & 13 & 2,930032 & $0,003390^{*}$ \\
\hline & \multirow{6}{*}{ Esquerda } & 9.000 & 26 & 13 & 1,346772 & 0,178055 \\
\hline & & 10.000 & 26 & 13 & 1,450503 & 0,146919 \\
\hline & & 11.000 & 26 & 13 & 1,566168 & 0,117310 \\
\hline & & 12.500 & 26 & 13 & 1,917599 & 0,055163 \\
\hline & & 14.000 & 26 & 13 & 2,771530 & $0,005580^{*}$ \\
\hline & & 16.000 & 26 & 13 & 2,847092 & $0,004412^{*}$ \\
\hline \multirow{12}{*}{40 a 51 anos $(n=30)$} & \multirow{6}{*}{ Direita } & 9.000 & 15 & 15 & 0,178808 & 0,858088 \\
\hline & & 10.000 & 15 & 15 & 0,285910 & 0,774947 \\
\hline & & 11.000 & 15 & 15 & 0,197617 & 0,843345 \\
\hline & & 12.500 & 15 & 15 & 1,357304 & 0,174686 \\
\hline & & 14.000 & 15 & 15 & 2,166932 & $0,030241^{*}$ \\
\hline & & 16.000 & 15 & 15 & 1,651194 & 0,098700 \\
\hline & \multirow{6}{*}{ Esquerda } & 9.000 & 15 & 15 & $-0,089174$ & 0,928944 \\
\hline & & 10.000 & 15 & 15 & 1,039544 & 0,298553 \\
\hline & & 11.000 & 15 & 15 & 1,472249 & 0,140955 \\
\hline & & 12.500 & 15 & 15 & 1,708635 & 0,087520 \\
\hline & & 14.000 & 15 & 15 & 1,881216 & 0,059943 \\
\hline & & 16.000 & 15 & 15 & 2,358893 & $0,018330^{*}$ \\
\hline
\end{tabular}

${ }^{*}$ Valores significativos $(\mathrm{p} \leq 0,05)-$ Teste $t$

Tabela 5. Demonstrativo da média dos limiares de altas frequências em sujeitos, de ambos os grupos, com limiares auditivos convencionais normais $(n=77)$

\begin{tabular}{|c|c|c|c|c|c|c|}
\hline \multirow{2}{*}{ Orelha } & \multirow{2}{*}{ Frequência $(\mathrm{Hz})$} & \multicolumn{2}{|c|}{ Expostos } & \multicolumn{2}{|c|}{ Não expostos } & \multirow[b]{2}{*}{ Valor $p$} \\
\hline & & Média & $\mathrm{DP}$ & Média & DP & \\
\hline \multirow{6}{*}{ Direita } & 9.000 & 17,6 & 18,8 & 13,1 & 9,8 & 0,1688 \\
\hline & 10.000 & 20,1 & 10,7 & 20,7 & 14,0 & 0,8412 \\
\hline & 11.200 & 18,5 & 10,1 & 19,2 & 16,6 & 0,8092 \\
\hline & 12.500 & 26,8 & 17,0 & 28,5 & 20,9 & 0,6898 \\
\hline & 14.000 & 31,4 & 16,1 & 22,4 & 18,3 & $0,0294^{*}$ \\
\hline & 16.000 & 35,3 & 17,2 & 23,3 & 19,6 & $0,0093^{\prime}$ \\
\hline \multirow{6}{*}{ Esquerda } & 9.000 & 14,4 & 7,1 & 14,4 & 10,1 & 0,9757 \\
\hline & 10.000 & 16,8 & 9,1 & 19,9 & 14,2 & 0,2515 \\
\hline & 11.200 & 18,1 & 11,3 & 20,8 & 15,7 & 0,3731 \\
\hline & 12.500 & 26,4 & 13,4 & 29,1 & 17,6 & 0,4575 \\
\hline & 14.000 & 30,4 & 17,7 & 24,5 & 17,0 & 0,1455 \\
\hline & 16.000 & 31,4 & 16,3 & 25,4 & 18,9 & 0,1865 \\
\hline
\end{tabular}

${ }^{*}$ Valores significativos $(\mathrm{p} \leq 0,05)-$ Teste $t$

Legenda: DP = desvio-padrão 
nessas proporções elevadas, porém, observaram entalhe acústico nas frequências de 4.000 ou $6.000 \mathrm{~Hz}$, em maior ocorrência do que o esperado quando comparados a indivíduos não músicos, considerando-se a mesma idade e gênero ${ }^{(1,3,8,9,18-20)}$.

Observou-se que os limiares de audibilidade dos músicos foram piorando em função do tempo de exposição à música e, neste grupo estudado, quanto maior o tempo de exposição à música (em anos), piores foram os limiares auditivos encontrados pela audiometria de altas frequências (Tabela 1). As diferenças entre os limiares de audibilidade e o tempo de exposição à música foram mais evidentes no grupo exposto há mais de 21 anos. Porém, para os limiares auditivos nas frequências $12.500 \mathrm{~Hz}$ (ambas as orelhas), $10.000 \mathrm{~Hz}$ (orelha direita) e $14.000 \mathrm{~Hz}$ (orelha esquerda), as diferenças foram evidenciadas com um menor tempo de exposição à música (11 a 20 anos). De acordo com outro estudo ${ }^{(21)}$, as exposições à música em níveis elevados, como em bandas e orquestras sinfônicas, acarretam alterações auditivas tanto quanto aquelas encontradas em trabalhadores expostos ao ruído intenso de indústrias. Porém, o que se observou com esse estudo é que os efeitos da música foram mais perceptíveis após um tempo de exposição maior que 21 $a_{n o s}{ }^{(19)}$. Já entre os trabalhadores expostos ao ruído intenso de indústrias, as perdas auditivas são observadas depois de cinco a dez anos de exposição a esse ruído ${ }^{(10)}$.

$\mathrm{Na}$ comparação dos limiares auditivos entre os grupos (Tabela 2), observou-se um decréscimo da sensibilidade auditiva a partir de $9.000 \mathrm{~Hz}$ em ambos os grupos. A literatura na área justifica esta situação devido ao fato de que no processo auditivo há necessidade de maior energia sonora para detectar sons agudos ${ }^{(21,22)}$. Porém, na comparação dos limiares tonais pela audiometria de altas frequências entre os grupos, observou-se que os músicos apresentaram limiares auditivos piores do que o grupo não exposto, para as frequências de 14.000 e $16.000 \mathrm{~Hz}$ em ambas as orelhas e em $9.000 \mathrm{~Hz}$ à direita. Um estudo que analisou 187 sujeitos expostos ao ruído ocupacional e 52 sujeitos não expostos ao ruído, referiu que no grupo exposto ao ruído, os limiares auditivos tonais por audiometria de altas frequências foram mais elevados que os do grupo não exposto ao ruído, sendo mais acentuada a diferença nos limiares tonais da frequência de $14.000 \mathrm{~Hz}^{(23)}$. Em outro estudo $^{(24)}$, também foi encontrado um decréscimo dos limiares auditivos tonais em ambas as orelhas conforme o aumento das altas frequências, sendo mais acentuada a partir de $14.000 \mathrm{~Hz}$. Outros autores ${ }^{20,25)}$ encontraram diferenças nos limiares auditivos entre grupos expostos e não expostos a níveis de pressão sonora elevados ocupacionais nas frequências de $12.500 \mathrm{~Hz}$ e $14.000 \mathrm{~Hz}$; observaram que a média dos limiares tonais pela audiometria de altas frequências estava pior quando comparada com a média dos limiares tonais até $8.000 \mathrm{~Hz}$, o que levou os autores a sugerirem um início de acometimento auditivo nas frequências altas (a partir de $8.000 \mathrm{~Hz}$ ) devido à exposição a níveis elevados de pressão sonora (música).

A audiometria de altas frequências é reconhecida por alguns autores ${ }^{(26-29)}$ como uma prática importante no monitoramento auditivo de sujeitos expostos a níveis elevados de pressão sonora, porém, nesse estudo, observou-se maior ocorrência de diferenças entre os grupos para os limiares de audibilidade nas frequências convencionais (Tabela 3) que nas altas frequências (três frequências com diferenças).

Alguns autores ${ }^{(14,30)}$ enfatizam também a necessidade de se controlar a variável idade na investigação de sujeitos expostos ao ruído quando se realiza a audiometria de altas frequências, pois a idade interfere nos limiares a partir de $8.000 \mathrm{~Hz}$, mais do que interfere nos limiares até $8.000 \mathrm{~Hz}$, nos quais a ação do ruído é predominante à ação da idade. A audiometria de altas frequências possibilita a percepção do desgaste auditivo causado pelo fator idade antes mesmo de aparecerem sinais na audiometria convencional ${ }^{(30)}$. No presente estudo, no entanto, além do desgaste natural pela idade há a exposição a níveis elevados de pressão sonora, o que modifica o padrão de alteração auditiva.

Então, quando os limiares auditivos foram analisados entre os grupos, controlada a idade pela aplicação da tabela I.S.O. 1999 (Tabela 3), ocorreram diferenças entre os grupos na maioria das frequências, em ambas as orelhas. E, quando se analisou os limiares auditivos pela audiometria de altas frequências, entre os grupos, separados pela faixa etária (Tabela 4), ocorreram diferenças entre os grupos a partir de 30 anos de idade. Para a faixa etária de 20 a 30 anos, não houve diferenças entre os limiares auditivos pela audiometria de altas frequências.

Para os sujeitos com idades entre 30 e 39 anos, os limiares auditivos no grupo exposto à música apresentaram-se piores em 14.000 e $16.000 \mathrm{~Hz}$ em ambas as orelhas; e na orelha direita, os limiares tonais nos músicos também foram piores em $9.000 \mathrm{~Hz}$.

Na faixa etária de 40 a 51 anos de idade os limiares auditivos dos sujeitos expostos à música foram piores para a frequência de $14.000 \mathrm{~Hz}$ na orelha direita e em $16.000 \mathrm{~Hz}$ na orelha esquerda, o que pode estar relacionado à exposição à música.

Para se verificar possíveis sinais precoces de lesões auditivas pela exposição à música, comparou-se os limiares auditivos nas altas frequências para os sujeitos com limiares tonais convencionais dentro dos padrões de normalidade, antes da aplicação da I.S.O. 1999 (Tabela 5). As diferenças nos limiares tonais, entre os grupos, foram nas frequências $14.000 \mathrm{~Hz}$ e $16.000 \mathrm{~Hz}$ na orelha direita. Em estudo com músicos apresentando audiogramas convencionais normais, mas com piora nos limiares por audiometria de altas frequências, observaram um entalhe acústico na frequência de $12.500 \mathrm{~Hz}$ bilateralmente e na frequência de $14.000 \mathrm{~Hz}$ na orelha direita ${ }^{(25)}$. Esses resultados podem sugerir um início de alteração auditiva devido à exposição a níveis de pressão sonora elevados.

\section{CONCLUSÃO}

Os limiares auditivos tonais do grupo de músicos (grupo exposto) apresentaram alterações auditivas permanentes, evidenciando diferenças em relação ao grupo não exposto à música profissionalmente, registradas na avaliação audiológica convencional e na audiometria de altas frequências.

Os resultados deste estudo demonstraram a necessidade de atuação para a promoção da saúde por meio dos Programas de Preservação Auditiva, envolvendo medidas de controle do som intenso (coletiva e individualmente), além de ações educativas para músicos profissionais. Sugere-se estudos 
que comparem o padrão de audibilidade entre músicos e trabalhadores expostos ao ruído.

* CGOG contribuiu com a elaboração do projeto, coleta de dados e análise, estruturação do texto do artigo; ABML colaborou com a coleta de dados e análise dos dados, revisão de bibliografia, revisão do texto do artigo; BSZ contribuiu com a coleta de dados, revisão de bibliografia, revisão do texto do artigo; JMM colaborou com a estruturação dos métodos, análise dos dados, revisão do texto do artigo; DL contribuiu com a coleta de dados, revisão de bibliografia, revisão do texto do artigo.

\section{REFERÊNCIAS}

1. Bride D, Gill F, Proops D, Harrington M, Gardiner K, Attwell C. Noise and the classical musician. BMJ. 1992;305(6868):1561-3.

2. Nataletti P, Sisto R, Pieroni A, Sanjus F, Annesi D. Pilot study of professional exposure and hearing functionality of orchestra musicians of a national lyric theatre. G Ital Med Lav Ergon. 2007;29(3 Suppl):496-8.

3. Emmerich E, Rudel L, Richter F. Is the audiologic status of professional musicians a reflection of the noise exposure in classical orchestral music? Eur Arch Otorhinolaryngol. 2008;265(7):753-8.

4. Royster JD, Royster LH, Killion MC. Sound exposures and hearing thresholds of symphony orchestra musicians. J Acoust Soc Am. 1991;89:2793-803.

5. Sataloff RT. Hearing loss in musicians. Am J Otol. 1991Mar;12(2):122-7.

6. Hellstrom PA, Axelsson A, Costa O. Temporary threshold shift induced by music. Scan Audiol Suppl. 1998;48:87-94.

7. Mendes MH, Morata TC, Marques JM. Acceptance of hearing protection aids in members of an instrumental and voice music band. Rev Bras Otorrinolaringol. 2007;73(6):785-92.

8. Santos L, Morata TC, Jacob LC, Albizu E, Marques JM, Paini M.. Music exposure and audiological findings in Brazilian disc jockeys (DJs). Int J Audiol. 2007;46(5):223-31.

9. Jansen EJ, Helleman HW, Dreschler WA, de Laat JA. Noise induced hearing loss and other hearing complaints among musicians of symphony orchestras. Int Arch Occup Environ Health. 2009Jan;82(2):153-64.

10. Goncalves CGO, Lacerda ABM, Zocoli AMF, Oliva FC, Almeida SB, Iantas MR. Percepção e o impacto da música na audição de integrantes de banda militar. Rev Soc Bras Fonoaudiol. 2009;14(4):515-20.

11. Dieroff HG, Schuhmann G, Meissner W, Barstsch R. Experiences with high-frequency hearing tests in the selection of personnel for noise occupations. Laryngorhinootologie. 1991;70:594-8.

12. Arnold DJ, Lonsbury-Martin BL, Martin GK. High-frequency hearing influences lower-frequency distortion-product otoacoustic emissions. Arch Otolaryngol Head Neck Surg. 1999;125(2):215-22.
13. Sahyeb DR, Costa Filho AO, Alvarenga KF. Audimetria de alta frequência: estudo com indivíduos audiologicamente normais. Rev Bras Otorrinolaringol. 2003;69(1):93-9.

14. Zeigelboim BS, Mangabeira-Albernaz PL, Fukuda Y. High frequency audiometry and chronic renal failure. Acta Otolaryngol. 2001;121(2):245-8.

15. Lopes AC, Otubo KA, Basso TC, Innocenti EJ, Marinelli JR, Pereira L. Perda auditiva ocupacional: audiometria tonal $\mathrm{X}$ audiometria de altas frequências. Arquivos Int. Otorrinolaringol. 2009;13(3):631-7.

16. Hoffman JS, Cunningham DR, Lorenz DJ. Auditory Thresholds and factors contributing to hearing loss in a large sample of percussionists. MPPA. 2006;21(2):47-47.

17. Phillips SL, Henrich VC, Mace ST. Prevalence of noise-induced hearing loss in student musicians. Int J Audiol. 2010;49(4):309-16.

18. Kaharit KR, Axelsson A, Hellstrom PA, Zachau G. Hearing assesment of classical orchestral musicians. Scand Audiol. 2001;30(1):13-23.

19. Morais D, Benito JL, Almaraz A. Acoustic trauma in classical music players. Acta Otorrinolaringol Esp. 2007;58(9):401-7.

20. Korres GS, Balatsouras DG, Tzagaroulakis A, Kandiloros D, Ferekidis E. Extende high-frequency audiometry in subjects exposed to occupational noise. B-ENT. 2008;4(3):147-55.

21. Zeigelboim BS, Oliveira VFBG, Marques JM, Jurkievicz AL. Limiares de audibilidade nas altas frequências em indivíduos de 20 a 30 anos com audição normal. Distúrb Comun. 2004;16(3):385-92.

22. Martinho T, Zeigelboim BS, Marques JM. Perfil Audiológico nas altas frequências em indivíduos de 30 a 40 anos com audição normal. Int. Arch. Otorrinolaringol. 2005;9(1):18-25.

23. Ahmed HO, Dennis JH, Badrant O, Ismail M, Ballal SG, Ashoor A, et al. High-frequency $(10-18 \mathrm{kHz})$ hearing thresholds: reliability and effects of age and occupacional noise exposure. Occup Med (Lond). 2001;51(4):245-58.

24. Fernandes JB, Mota HB. Estudo dos limiares de audibilidade nas altas frequências em trabalhadores expostos a ruído e solvente. Pro Fono. 2001;13(1):1-8.

25. Amorim RB, Lopes AC, Santos KTP, Melo ADP, Lauris JRP. Alterações auditivas da exposição ocupacional em músicos. Arquivos Int. Otorrinolaringol. 2008;12(3):377-83.

26. Hallmo P, Borchgevink HM, Mair IWS. Extended high-frequency thresholds in noise-induced hearing loss. Scand Audiol. 1995;24(1):47-52.

27. Castro IFC, Conde AC, Paiva AQF, Oliveira LTN, Bernardi APA. Estudo do perfil audiométrico em altas frequências em trabalhadores expostos ao ruído. Rev CEFAC. 2004;6(2):203-8.

28. Porto MAA, Gahyva DLC, Lauris JRC, Lopes AC. Avaliação da audição em frequências ultra-altas em indivíduos expostos ao ruído ocupacional. Pro Fono. 2004;16(3):237-50.

29. Somma G, Coppeta L, Magrini A, Parrella M, Cappelletti MC, Gardi S. Extended high frequency audiometry in the prevention of noise-induced hearing loss. G Ital Med Lav Ergon. 2007;29(suppl. 3):258-60.

30. Schochat E, Rabelo CM, Sanfins MD. Processamento auditivo central: testes tonais de padrão de frequência e duração em indivíduos normais de 7 a 16 anos de idade. Pro Fono. 2000;12(2):1-7. 